JOURNAL OF THE

AMERICAN MATHEMATICAL SOCIETY

Volume 24, Number 4, October 2011, Pages 999-1014

S $0894-0347(2011) 00702-9$

Article electronically published on April 8, 2011

\title{
DENSITY OF POSITIVE LYAPUNOV EXPONENTS FOR $\operatorname{SL}(2, \mathbb{R})$-COCYCLES
}

\author{
ARTUR AVILA
}

\section{INTRODUCTION}

The understanding of the frequency of hyperbolic behavior is one of the central themes in dynamics [BV]. Precise questions can be posed on several levels, for instance, in the context of area-preserving maps:

1. Under suitable smoothness assumptions, quasiperiodicity, and hence the absence of any kind of hyperbolicity, is non-negligible in a measure-theoretical sense [Kol] (under suitable smoothness assumptions).

2. In low regularity $\left(C^{1}\right)$, failure of non-uniform hyperbolicity (here nonuniform hyperbolicity can be understood as positivity of the metric entropy) is a fairly robust phenomenon in the topological sense [B].

On the other hand, very little is understood about the emergence of non-uniformly hyperbolic area-preserving diffeomorphisms "in the wild": indeed all known examples are either Anosov or very carefully cooked modifications of uniformly hyperbolic systems. While in low regularity one has so much flexibility to perturb that one can nevertheless show that positive metric entropy is dense in the $C^{1}$-topology (e.g., by embedding renormalized "cooked examples" away from Anosov map 1 1), whether this is true for reasonably smooth dynamics seems to be far beyond our current knowledge.

In this paper we consider the question of the density of non-uniform hyperbolicity in the considerably simpler context of $\mathrm{SL}(2, \mathbb{R})$-cocycles. Let $f: X \rightarrow X$ be a homeomorphism of a compact metric space, and let $\mu$ be a probability measure invariant by $f$. If $A: X \rightarrow \operatorname{SL}(2, \mathbb{R})$ is a continuous map, we can define the $\operatorname{SL}(2, \mathbb{R})$ cocycle $(f, A)$, acting on $X \times \mathbb{R}^{2}$, by $(x, y) \mapsto(f(x), A(x) \cdot y)$. The iterates of the cocycle have the form $\left(f^{n}, A_{n}\right)$, and for $n \geq 1$ we have $A_{n}(x)=A\left(f^{n-1}(x)\right) \cdots A(x)$.

Received by the editors May 25, 2010 and, in revised form, August 2, 2010, and March 22, 2011

2010 Mathematics Subject Classification. Primary 37H15.

This research was partially conducted during the period when the author was a Clay Research Fellow.

${ }^{1}$ In fact any area-preserving map can be approximated by one which is either Anosov or has an elliptic periodic point. In the latter case, it can be further perturbed so that some iterate of the dynamics is the identity on some disk. By further perturbation one can create small periodic disks with high period and isometric behavior, and a further perturbation allows one to realize, in a small scale, any given smooth area-preserving map of the unit disk (essentially by writing it as a composition of many smooth area-preserving maps close to the identity), thus in particular an example with positive metric entropy $\mathrm{Ka}$.

(C)2011 American Mathematical Society Reverts to public domain 28 years from publication 
The Lyapunov exponent is defined by

$$
L(A)=L(f, \mu, A)=\lim _{n \rightarrow \infty} \frac{1}{n} \int \ln \left\|A_{n}(x)\right\| d \mu(x) .
$$

The Lyapunov exponent is always non-negative, and a most fundamental dynamical property of the cocycle $(f, A)$ is whether it is non-zero. Examples of $\mathrm{SL}(2, \mathbb{R})$ cocycles include the tangent dynamics $(f, D f)$ of a $C^{1}$ conservative map of the torus $f: \mathbb{R}^{2} / \mathbb{Z}^{2} \rightarrow \mathbb{R}^{2} / \mathbb{Z}^{2}$ (in this case the Lyapunov exponent coincides with the metric entropy), but in the cocycle setting we have the flexibility of "perturbing $D f$ independently of $f "$, which considerably simplifies the analysis.

Problem 1.1. Can a cocycle with zero Lyapunov exponent be approximated by one with a positive Lyapunov exponent?

As usual in the cocycle setting, we take the point of view that the base dynamics (given by $f$ and $\mu$ ) should be regarded as fixed and only the fibered dynamics (given by $A$ ) should be allowed to vary. As mentioned before, the issue of regularity is of course usually important in such approximation questions. For instance, though we described a general setting above, when $f: X \rightarrow X$ is, say, a smooth diffeomorphism of a smooth manifold, one often wants to restrict considerations to smooth $A$, and correspondingly one wishes to obtain approximations that are close as smooth maps.

Obviously high regularity density results are a priori harder to obtain than low regularity ones. Indeed in the lowest regularity, the continuous category, Problem 1.1 can be easily resolved using Kotani theory: positive Lyapunov exponents are dense if and only if $f$ is not periodic on $\operatorname{supp} \mu$ (that is, $f^{k} \mid \operatorname{supp} \mu \neq$ id for every $k \geq 1$ ); see Lemma 3.1 .

Of course the continuous category allows for somewhat drastic perturbations, and closely related problems have different answers in low and high regularity. For instance, if $f$ is a Bernoulli shift, then there exist Hölder cocycles with positive Lyapunov exponent which can be approximated by continuous cocycles with zero Lyapunov exponent, but not by a Hölder one 2 In fact the analysis of high regularity has generally been restricted so far to specific subclasses of dynamical systems (for instance, with hyperbolic [V] or quasiperiodic behavior [FK], [A1]).

In this paper we will give a complete solution to Problem 1.1; we will both treat arbitrary dynamics (as defined above) and all usual regularity classes (e.g., Hölder, Sobolev, smooth, Gévrey, analytic). To describe precisely what we have in mind as a regularity class, it is convenient to introduce the following concept. A topological space $\mathfrak{B}$ continuously included in $C(X, \mathrm{SL}(2, \mathbb{R}))$ is ample if there exists some dense vector space $\mathfrak{b} \subset C(X, \operatorname{sl}(2, \mathbb{R})$ ), endowed with some finer (than uniform) topological vector space structure, such that for every $A \in \mathfrak{B}, e^{b} A \in \mathfrak{B}$ for every $b \in \mathfrak{b}$, and the map $b \mapsto e^{b} A$ from $\mathfrak{b}$ to $\mathfrak{B}$ is continuous. Notice that if $X$ is a compact smooth or analytic manifold, then the usual spaces of smooth or analytic maps $X \rightarrow \mathrm{SL}(2, \mathbb{R})$ are ample in our sense.

Theorem 1. Assume that $f$ is not periodic on $\operatorname{supp} \mu$, and let $\mathfrak{B} \subset C(X, \mathrm{SL}(2, \mathbb{R}))$ be ample. Then the Lyapunov exponent is positive for a dense subset of $\mathfrak{B}$.

\footnotetext{
${ }^{2}$ The Hölder exponent $\alpha>0$ being fixed. This happens for instance if $f$ has two fixed points $p$ and $q$ and $0<\operatorname{tr} A(p)<2<\operatorname{tr} A(q)<2+\epsilon(\alpha)$. See BGV].
} 
1.1. Schrödinger cocycles. The most studied subclass of $\mathrm{SL}(2, \mathbb{R})$-cocycles is the class of Schrödinger cocycles, when

$$
A(x)=A^{(E-v)}(x)=\left(\begin{array}{cc}
E-v(x) & -1 \\
1 & 0
\end{array}\right)
$$

for some $E \in \mathbb{R}, v \in C(X, \mathbb{R})$. Those appear naturally in the analysis of the Schrödinger operators of the form

$$
(H u)_{n}=u_{n+1}+u_{n-1}+v\left(f^{n}(x)\right) u_{n},
$$

since an eigenfunction $H u=E u$ satisfies $A_{n}^{(E-v)}(x)\left(\begin{array}{c}u_{0} \\ u_{-1}\end{array}\right)=\left(\begin{array}{c}u_{n} \\ u_{n-1}\end{array}\right)$. It will be convenient to use the short-handed notation $L(v)=L\left(A^{(v)}\right)$ for the Lyapunov exponent of Schrödinger cocycles.

The proof of Theorem 1 can be easily adapted to show:

Theorem 2. Assume that $f$ is not periodic on supp $\mu$, and let $V \subset C(X, \mathbb{R})$ be a dense vector space endowed with a finer topological vector space structure. Then for every $E \in C(X, \mathbb{R})$, the set of $v \in V$ such that $L(E-v)>0$ is dense.

Taking $E$ constant in Theorem 2, we see that for each fixed energy $E$ the Lyapunov exponent is positive for a dense set of potentials $v$. However, since in the analysis of the Schrödinger operator we need to vary the energy for some fixed potential, it is natural to wonder whether the set of potentials for which the Lyapunov exponent is positive for a dense subset of energies is dense in $V$, and if so, whether it is "large".

Under the mild additional assumption that $V$ is a Baire space, it is clear that Theorem 2 yields the following: for a generic set (thus large topologically) of $v \in V$, the Lyapunov exponent is positive for a dense subset of energies.

However, it is often the case that a large set in the topological sense is not large in the measure-theoretic sense; hence it makes sense to ask whether this is what is going on here. Of course we first need a notion of "measure-theoretically large": a common such notion is provided by the concept of prevalence in a separable Banach space $V$ : a subset $P \subset V$ is said to be prevalent if there exists a probability measure $\nu$ with compact support in $V$ such that for every $v \in V$ and for almost every $w$ with respect to $\nu$ we have $v+w \in P$. This notion coincides with "full Lebesgue measure" in finite dimensional settings; see [HSY].

Theorem 3. Assume that $f$ is not periodic on $\operatorname{supp} \mu$, and let $V \subset C(X, \mathbb{R})$ be a dense vector space endowed with a finer separable Banach space structure. Then the set of $v \in V$ such that $L(E-v)>0$ for a dense set of $E \in \mathbb{R}$ is prevalent.

Remark 1.2. The dense set of energies with a positive Lyapunov exponent provided by Theorem 3 is necessarily "non-negligible everywhere" in the sense that it intersects any open set in a positive measure set. Indeed, while the Lyapunov exponent is not always a continuous function of $E$, it is measurably continuous everywhere in the sense that for every $E_{0} \in \mathbb{R}$ and for every $\epsilon>0, E_{0}$ is a Lebesgue density point of the set $\left\{\left|L(E-v)-L\left(E_{0}-v\right)\right|<\epsilon\right\}$. This can be obtained right away from the Thouless formula representation $L(E-v)=\int \ln \left|E^{\prime}-E\right| d N\left(E^{\prime}\right)$, see e.g. [AS, where $N$ (known as the integrated density of states) is the distribution of a compactly supported and continuous probability measure on $\mathbb{R}$. 
1.2. Further comments. As mentioned above, previous progress on Problem 1.1 , in high regularity, was restricted to specific classes of dynamical systems. Two important classes of examples are systems with periodic orbits of arbitrarily large periods (allowing one to deal with situations arising in non-uniformly hyperbolic dynamics) and systems whose behavior is sufficiently close to quasiperiodic. In both cases, the most important techniques are provided by some form of Kotani theory [ $\mathrm{K}$, which imposes strict constrains on the dynamics of cocycles with stably zero Lyapunov exponents. Even when successful, the results were often highly dependent on the regularity: for translations of tori, density was proved in the analytic category, with the inductive topology (which is not Baire), but not in Banach spaces of analytic functions [A1] for the skew-shift, the smooth category could be covered, but not the analytic one; etc. In this paper, we take a completely different approach that will allow us to bypass the detailed understanding of the basis dynamics, relying instead on a simple general property of the dependence of the Lyapunov exponent with respect to parameters which we have discovered.

While the Lyapunov exponent is generally a rather wild function of parameters (see [B]), we will show that it can be manipulated to yield regularized expressions with a nice dependence. Perhaps the simplest example of a regularizing expression involving the Lyapunov exponent is the $\mathrm{AB}$ formula

$$
\int_{0}^{1} L\left(A R_{\theta}\right) d \theta=\int \ln \frac{\|A\|+\|A\|^{-1}}{2} d \mu
$$

Here $R_{\theta}$ is the rotation of angle $2 \pi \theta$. In [AK, other expressions for regularizing the Lyapunov exponent have been shown to exist; however all of them had an intrinsic non-local nature: to obtain good dependence with respect to parameters, one combined the Lyapunov exponents of "topologically non-trivial" families of cocycles of a suitable type (e.g., perturbations of $\theta \mapsto A R_{\theta}$ ). This obviously makes such formulas unsuitable to address approximation problems, which are intrinsically local (while (4) easily implies that for most $A$ there exist some $\theta$ such that $L\left(A R_{\theta}\right)>$ 0 , it gives no information on their location).

In this paper we produce the first local regularization expressions for the Lyapunov exponent with analytic dependence on a parameter. Let us describe one such expression which is of use for the Schrödinger case. Denote by $\|\cdot\|$ the sup norm in $C(X, \mathbb{R})$ and for $r>0$ let $\mathcal{B}(r)$ denote the open ball $\{\|w\|<r\}$ in $C(X, \mathbb{R})$.

Theorem 4. For every $\epsilon>0$, for every $v \in C(X, \mathbb{R})$,

$$
\Phi_{\epsilon}(v, w)=\int_{-1}^{1} \frac{1-t^{2}}{\left|t^{2}+2 i t+1\right|^{2}} L\left(v+\epsilon\left(t+\left(1-t^{2}\right) w\right)\right) d t
$$

defines an analytic function of $w$ in $\mathcal{B}\left(2^{-3 / 2}\right)$.

With such a kind of result in hand, we can easily extend the well-known density of positive Lyapunov exponents in the weakest regularity (continuous category) to arbitrary regularity.

Let us note also that our methods can be applied to study the prevalence of density (in energy) of positive Lyapunov exponents for specific parametrized families of 
potentials. The following result (related to the proof of Theorem 3) demonstrates this:

Theorem 5. Let $J \subset C(X, \mathbb{R})$ be a segment such that $L(E-v)>0$ is dense in $(E, v) \in \mathbb{R} \times J$. Then for almost every $v \in J$, there exists a dense set of $E \in \mathbb{R}$ such that $L(E-v)>0$.

This result is an immediate consequence of a more quantitative estimate; see Theorem 9

Let us conclude with some comments on the related issue of prevalence of positive Lyapunov exponents: could it be possible that positive Lyapunov exponents would be not merely dense, but measure-theoretically typical? This is well known not to be the case: for smooth or analytic quasiperiodic cocycles with suitable Diophantine conditions on the frequency, KAM theory (first advanced in this context by Dinaburg and Sinai [DS] implies that zero Lyapunov exponents are non-negligible (e.g., in the Kolmogorov sense: they do appear with positive probability in open sets of parametrized families). However, in other settings (for instance, in the presence of some hyperbolicity and regularity; see $[\mathbf{V}]$ ) it has been established that zero Lyapunov exponents are indeed negligible.

More generally, Kotani theory severely restricts the behavior of typical cocycles with zero Lyapunov exponent (e.g., iterates not only grow subexponentially but stay bounded in the $L^{1}$-sense). Other such considerations eventually led to the formulation (see, e.g., [D, J]) of the far-reaching Kotani-Last Conjecture (for Schrödinger cocycles): except for almost periodic potentials, the Lyapunov exponent should be positive for almost every energy. Unfortunately this conjecture was recently shown to be false $\underline{\mathrm{A} 3}$, and currently no reasonable replacement has been advanced.

1.3. Outline of the paper. We start most arguments with the analysis of the case of Schrödinger cocycles, which is slightly more straightforward. In Section 2 we give a proof of analyticity of a regularized expression, Theorem 6. which is slightly more general than Theorem 4 , and then provide a non-Schrödinger version, Theorem 7 In Section 3 we go on to prove Theorems 2 and 1, using analyticity to make the transition from local to infinitesimal information and in the process allowing us to jump from the easy continuous category to arbitrary regularity. The slightly more technical analysis of prevalence, Theorem 3, is carried out in Section 4.

\section{Regularizing the parameter Dependence of the Lyapunov exponent}

In this section we will prove the following more general version of Theorem 4 .

Theorem 6. For every $\epsilon>0$, for every $v, v_{0} \in C(X, \mathbb{R})$, with $\eta=\inf v_{0}>0$,

$$
\Phi_{\epsilon}\left(v, v_{0}, w\right)=\int_{-1}^{1} \frac{1-t^{2}}{\left|t^{2}+2 i t+1\right|^{2}} L\left(v+\epsilon\left(v_{0}+\left(1-t^{2}\right) w\right)\right) d t
$$

defines an analytic function of $w$ in the ball $\mathcal{B}\left(\eta / 2^{3 / 2}\right)$. Moreover, $w \mapsto \Phi_{\epsilon}\left(v, v_{0}, w\right)$ depends continuously (as an analytic function) on $v$. 
2.1. $\mathrm{SL}(2, \mathbb{C})$-cocycles and uniform hyperbolicity. We will need a few basic facts about the dynamics of $\mathrm{SL}(2, \mathbb{C}$ )-cocycles (all results discussed below are well known, with some proofs given for the convenience of the reader). Similarly to $\mathrm{SL}(2, \mathbb{R})$-cocycles, given $A \in C(X, \mathrm{SL}(2, \mathbb{C}))$, one defines the $\mathrm{SL}(2, \mathbb{C})$-cocycle $(f, A)$ acting on $X \times \mathbb{C}^{2}$ by $(f, A):(x, w) \mapsto(f(x), A(x) \cdot w)$. We use the same notation for the iterates $(f, A)^{n}=\left(f^{n}, A_{n}\right)$ and define the Lyapunov exponent $L(A)$ as before, by (11).

Naturally the cocycle $(f, A)$ induces an action on $X \times \mathbb{P C}^{2}$, which we will not distinguish notationally. Below we will use the spherical metric in $\mathbb{P C}^{2}$.

We call $A \in C(X, \mathrm{SL}(2, \mathbb{C}))$ uniformly hyperbolic if there exist $C>0, \lambda>1$ such that $\left\|A_{n}(x)\right\| \geq C \lambda^{n}$ for every $x$. This is equivalent (see, e.g., $[\mathrm{Y}]$ ) to the existence of a pair of functions (the unstable and stable directions) $u=u_{A}$ and $s=s_{A}$ in $C\left(X, \mathbb{P C}^{2}\right)$ such that there exist $C_{A}>0, \lambda_{A}>1$ such that $A(x) \cdot u(x)=u(f(x))$, $A(x) \cdot s(x)=s(f(x)),\left\|A_{n}(x) \cdot z\right\|,\left\|A_{-n}(x) \cdot w\right\| \leq C_{A} \lambda_{A}^{-n}$ for every $n \geq 1$ and all unit vectors $z \in s(x), w \in u(x)$. Notice that the unstable and stable directions are uniquely defined by those properties. Notice that necessarily $u(x) \neq s(x)$ for every $x \in X$.

A conefield is an open set $U \subset X \times \mathbb{P C}^{2}$ of the form $\bigcup_{x \in X}\{x\} \times U_{x}$ with $\varnothing \neq \bar{U}_{x} \neq$ $\mathbb{P C}^{2}$. It is easy to see that if $m \in \mathbb{P C}^{2}$ does not coincide with the stable direction at $x$, then the distance between $A_{n}(x) \cdot m$ and $u\left(f^{n}(x)\right)$ decays exponentially fast, with the leading constant depending only on a lower bound on the distance between $s(x)$ and $m$. In particular, if $U$ is a conefield and $(x, u(x)) \in U,(x, s(x)) \notin \bar{U}$ for every $x \in X$, then there exists $n \geq 1$ such that $\left(f^{n}(x), A_{n}(x) \cdot m\right) \in U$ for every $(x, m) \in \bar{U}$.

Conversely, uniform hyperbolicity can be detected by a conefield criterion: there exists a conefield $U$ and $n \geq 1$ such that $\left(f^{n}(x), A_{n}(x) \cdot m\right) \in U$ for every $(x, m) \in \bar{U}$ if and only if $A$ is uniformly hyperbolic (to get the direct implication, notice that the Schwarz lemma implies that $A_{n}(x): U_{x} \rightarrow U_{f^{n}(x)}$ strictly contracts the Poincaré metric, uniformly in $x$, which readily gives exponential growth of $\left.\left\|A_{k}\right\|\right)$. Notice that in this case we necessarily have $(x, u(x)) \in U$ and $(x, s(x)) \notin \bar{U}$ for every $x \in X$.

Let $\mathcal{U H} \subset C(X, \mathrm{SL}(2, \mathbb{C}))$ denote the set of uniformly hyperbolic $A$. By the conefield criterion, it is clear that $\mathcal{U H}$ is an open set.

Lemma 2.1. For every $x \in X, A \mapsto u_{A}(x)$ is a holomorphic function of $A \in \mathcal{U H}$.

Proof. Fix $A \in \mathcal{U H}$ and let $\epsilon_{0}$ be the infimum of the distance between $u_{A}(x)$ and $s_{A}(x), x \in X$. Fix $0<\epsilon<\epsilon_{0} / 2$, and let $U$ be the conefield consisting of all $(x, m)$ such that $m$ is $\epsilon$-close to $u(x)$. Let $n \geq 1$ be such that $\left(f^{n}(x), A_{n}(x) \cdot m\right) \in U$ for every $(x, m) \in \bar{U}$. Let $\mathcal{V} \subset C(X, \mathrm{SL}(2, \mathbb{C}))$ be the set of all $A^{\prime}$ such that $\left(f^{n}(x), A_{n}^{\prime}(x) \cdot m\right) \in U$ for every $(x, m) \in \bar{U}$. Then $\mathcal{V} \subset \mathcal{U H}$, and it is an open neighborhood of $A$.

For $A^{\prime} \in \mathcal{V}, u_{A^{\prime}}(x)$ is the limit of $u_{A^{\prime}}^{k}(x) \equiv A_{k n}^{\prime}\left(f^{-k n}(x)\right) \cdot u_{A}(x)$. Notice that for each $k \geq 1, A^{\prime} \mapsto u_{A^{\prime}}^{k}(x)$ is a holomorphic function taking values in the hemisphere of $\mathbb{P C}^{2}$ centered on $u_{A}(x)$. By Montel's theorem, the limiting function $A^{\prime} \mapsto u_{A^{\prime}}(x)$ is holomorphic.

Remark 2.2. It follows from the proof of the previous lemma (take $\epsilon>0$ arbitrarily small) that if $A \in \mathcal{U H}$ and $A^{\prime}$ is close to $A$, then $u_{A^{\prime}}$ is uniformly close to $u_{A}$. By 
the symmetric argument, $s_{A^{\prime}}$ is also uniformly close $s_{A}$. In particular, the distance between $u_{A^{\prime}}(x)$ and $s_{A^{\prime}}(x)$ is minorated for $A^{\prime}$ near $A$.

It is easy to see that the Lyapunov exponent is given by

$$
L(A)=\int_{X} \lambda\left(A(x), u_{A}(x)\right) d \mu=-\int_{X} \lambda(A(x), s(x)) d \mu,
$$

where we define the expansion coefficient of the matrix $A \in \mathrm{SL}(2, \mathbb{C})$ in the direction $m \in \mathbb{P} \mathbb{C}^{2}$ by $\lambda(A, m)=\ln \|A(x) \cdot z\|, z \in m$ a unit vector.

Lemma 2.3. The Lyapunov exponent $A \mapsto L(A)$ is a plurisubharmonic function of $A \in C(X, \mathrm{SL}(2, \mathbb{C}))$. Moreover, restricted to the set of uniformly hyperbolic $A$, it is a pluriharmonic function.

Proof. Plurisubharmonicity is immediate from the alternative expression (a consequence of subadditivity) of the Lyapunov exponent as the limit of the decreasing sequence of subharmonic functions $\int_{X} \ln \left\|A_{2^{n}}\right\|_{\mathrm{HS}} d \mu / 2^{n}$ (with $\|\cdot\|_{\mathrm{HS}}$ denoting the Hilbert-Schmidt norm).

Let $A \in \mathcal{U H}$. Let $h, v \in \mathbb{P C}^{2}$ be horizontal and vertical directions, and let $m \in \mathbb{P C}^{2} \backslash\{h, v\}$ be any other direction. Let $B: X \rightarrow \mathrm{SL}(2, \mathbb{C})$ be a bounded Borel function such that $B(x) \cdot u_{A}(x)=h$ and $B(x) \cdot s_{A}(x)=v$. Choose a small open neighborhood $\mathcal{V} \subset \mathcal{U H}$ of $A$, and let $\mathcal{B}: \mathcal{V} \times X \rightarrow \mathrm{SL}(2, \mathbb{C})$ be such that $\mathcal{B}\left(A^{\prime}, x\right) \cdot u_{A^{\prime}}(x)=h, \mathcal{B}\left(A^{\prime}, x\right) \cdot s_{A^{\prime}}(x)=v$ and $\mathcal{B}\left(A^{\prime}, x\right) \cdot B(x)^{-1} \cdot m=m$ and such that $\mathcal{B}\left(A^{\prime}, x\right)$ is a continuous function of $A^{\prime}$ (the directions $u_{A^{\prime}}, s_{A^{\prime}}, B^{-1} \cdot m$ remain away from each other by Remark 2.2). Then $\mathcal{B}\left(A^{\prime}, x\right)$ is a bounded Borel function of $\left(A^{\prime}, x\right)$ and it is holomorphic in $A^{\prime}$. Notice that $\mathcal{B}\left(A^{\prime}, f(x)\right) A^{\prime}(x) \mathcal{B}\left(A^{\prime}, x\right)^{-1}$ is diagonal, so it can be written as $\left(\begin{array}{cc}\gamma\left(A^{\prime}, x\right) & 0 \\ 0 & \gamma\left(A^{\prime}, x\right)^{-1}\end{array}\right)$, where $\gamma: \mathcal{V} \times X \rightarrow \mathbb{C} \backslash\{0\}$ is a Borel function bounded away from 0 and $\infty$ and $A^{\prime} \mapsto \gamma\left(A^{\prime}, x\right)$ is holomorphic for each $x \in X$. We will show that

$$
L\left(A^{\prime}\right)=\int_{X} \ln \left|\gamma\left(A^{\prime}, x\right)\right| d \mu(x)
$$

which implies that $A^{\prime} \mapsto L\left(A^{\prime}\right)$ is pluriharmonic in $\mathcal{V}$ (since for each $x \in X$, $A^{\prime} \mapsto \ln \left|\gamma\left(A^{\prime}, x\right)\right|$ is a bounded pluriharmonic function) $)$.

Let $\gamma_{n}\left(A^{\prime}, x\right)=\prod_{k=0}^{n-1} \gamma\left(A^{\prime}, f^{k}(x)\right)$. Then

$$
\mathcal{B}\left(A^{\prime}, f^{n}(x)\right) A_{n}^{\prime}(x) \mathcal{B}\left(A^{\prime}, x\right)^{-1}=\left(\begin{array}{cc}
\gamma_{n}\left(A^{\prime}, x\right) & 0 \\
0 & \gamma_{n}\left(A^{\prime}, x\right)^{-1}
\end{array}\right) .
$$

We have $\left|\gamma_{n}\left(A^{\prime}, x\right)\right| \geq C_{A^{\prime}}^{-1} \lambda_{A^{\prime}}^{n} \sup \|\mathcal{B}\|^{2}$, so for $n$ sufficiently large (independent of $x),\left|\gamma_{n}\left(A^{\prime}, x\right)\right|>1$. Thus

$$
\ln \left|\gamma_{n}\left(A^{\prime}, x\right)\right|-\ln \sup \|\mathcal{B}\|^{2} \leq \ln \left\|A_{n}^{\prime}(x)\right\| \leq \ln \left|\gamma_{n}\left(A^{\prime}, x\right)\right|+\ln \sup \|\mathcal{B}\|^{2},
$$

and we get

$$
L\left(A^{\prime}\right)=\lim \frac{1}{n} \int_{X} \ln \left|\gamma_{n}\left(A^{\prime}, x\right)\right| d \mu(x),
$$

and since $\int_{X} \ln \left|\gamma_{n}\left(A^{\prime}, x\right)\right| d \mu(x)=n \int_{X} \ln \left|\gamma\left(A^{\prime}, x\right)\right| d \mu(x)$, (8) follows.

The following gives a simple sufficient condition for uniform hyperbolicity of Schrödinger cocycles.

Lemma 2.4. If $v \in C(X, \mathbb{C})$ and $\Im v>0$, then $A^{(v)}$ is uniformly hyperbolic. 
Proof. Let $\mathcal{H}$ be the open hemisphere of $\mathbb{P} \mathbb{C}^{2}$ centered on the line through $\left(\begin{array}{l}i \\ 1\end{array}\right)$. Then $A^{(v)}(x) \cdot m \in \mathcal{H}$ for every $m \in \overline{\mathcal{H}}$ which is not vertical, and $A^{(v)}(x)$ takes the vertical direction to the horizontal direction. Thus $A_{2}^{(v)}(x) \cdot m \in \mathbb{H}$ for every $m \in \overline{\mathcal{H}}$. Thus the conefield criterion is verified, with $U=X \times \mathcal{H}$ and $n=2$.

2.2. Proof of Theorem 6. We extend the notation $\|\cdot\|$ to denote the sup norm in $C(X, \mathbb{C})$ (as well as in $C(X, \mathbb{R})$ as previously defined), and for $r>0$ we denote by $\mathcal{B}^{\mathbb{C}}(r)$ the open ball $\{\|w\|<r\}$ in $C(X, \mathbb{C})$.

Fix $v, v_{0} \in C(X, \mathbb{R})$ with $\eta=\inf v_{0}>0$, and for $z \in \mathbb{C}$ and $w \in C(X, \mathbb{C})$, let $\rho_{w}(z)=L\left(v+\epsilon z v_{0}+\epsilon^{2}\left(1-z^{2}\right) w\right)$.

If $t=\epsilon e^{2 \pi i \theta}$ with $0<\theta<1 / 2$ and $w \in \mathcal{B}^{\mathbb{C}}(\eta / 2 \epsilon)$, then

$$
\begin{aligned}
\Im\left(t v_{0}+\left(\epsilon^{2}-t^{2}\right) w\right) & \geq \epsilon \eta \sin 2 \pi \theta+\epsilon^{2} \Im\left(\left(1-e^{4 \pi i \theta}\right) w\right) \\
& \geq \epsilon\left(\eta \sin 2 \pi \theta-\epsilon\left|1-e^{4 \pi i \theta}\right||w|\right)=\epsilon \sin 2 \pi \theta(\eta-2 \epsilon|w|)>0 .
\end{aligned}
$$

It follows that for every $z \in \partial \mathbb{D} \cap \mathbb{H}$ (here $\mathbb{H}$ is the upper half-plane), $w \mapsto \rho_{w}(z)$ is pluriharmonic in $\mathcal{B}^{\mathbb{C}}(\eta / 2 \epsilon)$.

Notice that if $t=\epsilon\left(2^{1 / 2}-1\right) i$ and $w \in \mathcal{B}^{\mathbb{C}}\left(\eta / 2^{3 / 2} \epsilon\right)$, then

$$
\Im\left(t v_{0}+\left(\epsilon^{2}-t^{2}\right) w\right) \geq \epsilon \eta\left(2^{1 / 2}-1\right)+2^{3 / 2} \epsilon^{2}\left(2^{1 / 2}-1\right) \Im w>0 .
$$

It follows that for $z=\left(2^{1 / 2}-1\right) i, w \mapsto \rho_{w}(z)$ is a pluriharmonic function in the ball $\mathcal{B}^{\mathbb{C}}\left(\eta / 2^{3 / 2} \epsilon\right)$.

Let $\phi(z)=i(1-z) /(1+z)$ be the conformal map $\mathbb{D} \rightarrow \mathbb{H}$ which takes $(1, i,-1)$ to $(0,1, \infty)$. Then $\phi^{-1}(z)=-(z-i) /(z+i)$, and $\psi(z)=\phi^{-1}\left(\phi(z)^{1 / 2}\right)$ takes $\mathbb{D}$ to $\mathbb{D} \cap \mathbb{H}$. Notice that $\psi(0)=\left(2^{1 / 2}-1\right) i$.

It follows that

$$
\rho_{w}(\psi(0))-\int_{0}^{1 / 2} \rho_{w}\left(\psi\left(e^{2 \pi i \theta}\right)\right) d \theta
$$

is a pluriharmonic function on $\mathcal{B}^{\mathbb{C}}\left(\eta / 2^{3 / 2} \epsilon\right)$.

Assume now that $t=\delta e^{2 \pi i \theta}$ with $0<\theta<1 / 2,0<\delta<\epsilon$, and let us assume that $w \in \mathcal{B}(\eta / 2 \epsilon)$. Since $w$ is now assumed to be real valued, we get

$$
\Im\left(t v_{0}+\left(\epsilon^{2}-t^{2}\right) w\right) \geq \delta \sin 2 \pi \theta(\eta-2 \delta w \cos 2 \pi \theta)>0 .
$$

It follows that for every $w \in \mathcal{B}(\eta / 2 \epsilon), z \mapsto \rho_{w}(z)$ is harmonic through $\mathbb{D} \cap \mathbb{H}$. By the Poisson formula,

$$
\rho_{w}(\psi(0))=\int_{0}^{1} \rho_{w}\left(\psi\left(e^{2 \pi i \theta}\right)\right) d \theta
$$

whenever $w \in \mathcal{B}(\eta / 2 \epsilon)$.

We conclude that

$$
\int_{1 / 2}^{1} \rho_{w}\left(\psi\left(e^{2 \pi i \theta}\right)\right) d \theta
$$

is an analytic function on $w \in \mathcal{B}\left(\eta / 2^{3 / 2} \epsilon\right)$, since (14) and (17) agree in this region (by (16)) and (14) has a pluriharmonic extension. A simple computation shows 
that

$$
\Phi_{\epsilon}\left(v, v_{0}, \epsilon w\right)=\int_{-1}^{1} \frac{1-t^{2}}{\left|t^{2}+2 i t+1\right|^{2}} \rho_{w}(t) d t=\frac{\pi}{2} \int_{1 / 2}^{1} \rho_{w}\left(\psi\left(e^{2 \pi i \theta}\right)\right) d \theta,
$$

concluding the proof of analyticity.

For continuous dependence with respect to $v$, notice that $(v, w) \mapsto L\left(v+\epsilon z v_{0}+\right.$ $\left.\epsilon^{2}\left(1-z^{2}\right) w\right)$ is jointly continuous on $v \in C(X, \mathbb{R})$ and $w \in \mathcal{B}^{\mathbb{C}}\left(\eta / 2^{3 / 2} \epsilon\right)$, for every $z \in \partial \mathbb{D} \cap \mathbb{H}$ and also for $z=\left(2^{1 / 2}-1\right) i$ (again by uniform hyperbolicity), so that the pluriharmonic extension (14) of $w \mapsto \frac{2}{\pi} \Phi_{\epsilon}\left(v, v_{0}, \epsilon w\right)$ to $w \in \mathcal{B}^{\mathbb{C}}(\eta / 2 \epsilon)$ depends continuously on $v$ and $w$.

2.3. Non-Schrödinger case. Let $\|\cdot\|_{*}$ denote the sup norm in $C(X, \operatorname{sl}(2, \mathbb{R}))$ and $C(X, \operatorname{sl}(2, \mathbb{C}))$, and for $r>0$ let $\mathcal{B}_{*}(r)$ and $\mathcal{B}_{*}^{\mathbb{C}}(r)$ be the corresponding $r$-balls.

Theorem 7. There exists $\eta>0$ such that if $b \in C(X, \operatorname{sl}(2, \mathbb{R}))$ is $\eta$-close to $\left(\begin{array}{cc}0 & 1 \\ -1 & 0\end{array}\right)$, then for every $\epsilon>0$ and every $A \in C(X, \mathrm{SL}(2, \mathbb{R}))$,

$$
a \mapsto \int_{-1}^{1} \frac{1-t^{2}}{\left|t^{2}+2 i t+1\right|^{2}} L\left(e^{\epsilon\left(t b+\left(1-t^{2}\right) a\right)} A\right) d t
$$

is an analytic function of $a \in \mathcal{B}_{*}(\eta)$, which depends continuously (as an analytic function) on $A$.

Proof. The argument is mostly the same as for Theorem 6, so we just explain what needs to be modified. The key point is to find $\eta>0$ such that we can check the following:

1. For $z \in \partial \mathbb{D} \cap \mathbb{H}$ and for $z=\left(2^{1 / 2}-1\right) i, e^{\epsilon\left(z b+\left(1-z^{2}\right) a\right)} A$ is uniformly hyperbolic, provided $a \in \mathcal{B}_{*}^{\mathbb{C}}(\eta)$.

2. For $z \in \mathbb{D} \cap \mathbb{H}, e^{\epsilon\left(z b+\left(1-z^{2}\right) a\right)} A$ is uniformly hyperbolic, provided $a \in \mathcal{B}_{*}(\eta)$.

Once this is done, the remainder of the argument is unchanged.

Recall that $\mathcal{H}$ is the open hemisphere of $\mathbb{P C}^{2}$ centered at the line through $\left(\begin{array}{l}i \\ 1\end{array}\right)$. Its boundary consist of the real directions, $\partial \mathcal{H}=\mathbb{P R}^{2}$. Fix a direction $m \in \mathbb{P R}^{2}$, and let us consider the path $m_{\epsilon}=e^{\epsilon\left(z b+\left(1-z^{2}\right) a\right)} \cdot m \in \mathbb{P C}^{2}$. We claim that for $\eta>0$ small and for $z$ and $a$ as in either case 1 or 2 above, the derivative $\frac{d}{d \epsilon} m_{\epsilon}$ at $\epsilon=0$ points inside $\mathbb{H}$. This implies in particular that for every $\epsilon>0$ small, $e^{\epsilon\left(z b+\left(1-z^{2}\right) a\right)}$ takes $\overline{\mathcal{H}}$ into $\mathcal{H}$. By iteration, $e^{\epsilon\left(z b+\left(1-z^{2}\right) a\right)}$ takes $\overline{\mathcal{H}}$ into $\mathcal{H}$ for every $\epsilon>0$, and since any $\operatorname{SL}(2, \mathbb{R})$ matrix preserves $\mathcal{H}, e^{\epsilon\left(z b+\left(1-z^{2}\right) a\right)} A$ takes $\overline{\mathcal{H}}$ into $\mathcal{H}$ as well. Thus in either case 1 or 2 above, uniform hyperbolicity is verified through the conefield criterion, with $U=X \times \mathcal{H}$.

It remains to check the above derivative estimate. It will be convenient to identify $\mathbb{P C}^{2}$ with $\overline{\mathbb{C}}$, by taking the line through $\left(\begin{array}{l}x \\ y\end{array}\right)$ to $\frac{x}{y}$. Then $\mathcal{H}$ is identified with the upper half-plane $\mathbb{H}$.

Writing $b=\left(\begin{array}{cc}b_{1} & b_{2} \\ b_{3} & -b_{1}\end{array}\right), a=\left(\begin{array}{cc}a_{1} & a_{2} \\ a_{3} & -a_{1}\end{array}\right)$, we have

$$
\left.\frac{d}{d \epsilon} m_{\epsilon}\right|_{\epsilon=0}=z\left(2 b_{1} m+b_{2}-b_{3} m^{2}\right)+\left(1-z^{2}\right)\left(2 a_{1} m+a_{2}-a_{3} m^{2}\right) .
$$


Notice that in either case 1 or 2 above, $\left|\Im\left(1-z^{2}\right) a_{j}\right|=O(\eta \Im z), j=1,2,3$, which gives

$$
\left.\Im \frac{d}{d \epsilon} m_{\epsilon}\right|_{\epsilon=0}=\Im z\left(1+m^{2}\right)(1+O(\eta)),
$$

so choosing $\eta>0$ sufficiently small, $\frac{d}{d \epsilon} m_{\epsilon}$ has a positive imaginary component for $\epsilon=0$, so it points inside $\mathcal{H}$.

For the point ( $m=\infty$ in the above identification) missing from the above discussion, we can use a different identification taking the line through $\left(\begin{array}{l}x \\ y\end{array}\right)$ to $\frac{-y}{x}$, which takes $\mathcal{H}$ to $\mathbb{H}$ as before but identifies the missing point with 0 . Then we have

$$
\left.\frac{d}{d \epsilon} m_{\epsilon}\right|_{\epsilon=0}=-z b_{3}-\left(1-z^{2}\right) a_{3},
$$

which points inside $\mathcal{H}$ as before.

This establishes the desired claim.

\section{Density of POSITIVE LyAPUNOV EXPONENTS}

We assume throughout this section that $f \mid \operatorname{supp} \mu$ is not periodic.

Lemma 3.1. The set of $v \in C(X, \mathbb{R})$ such that $L(v)>0$ is dense.

Proof. Let $P \subset X$ be the set of periodic orbits of $f$.

If $\mu(P)<1$, a stronger result is provided by $\mathrm{AD}$ : for generic $v \in C(X, \mathbb{R})$, the set of $E \in \mathbb{R}$ such that $L(E-v)>0$ has full Lebesgue measure. (The results of [AD are stated for the most important case of $\mu$ ergodic, but the argument applies unchanged in this larger generality.)

Assume that $\mu(P)=1$. Below we will use well-known facts about periodic Schrödinger operators; see AMS] for a discussion and further references or A2 for a more recent discussion close in style to this paper. Let $P_{k} \subset X$ be the set of periodic orbits of period $k \geq 1$. Since $f \mid \operatorname{supp} \mu$ is not periodic, $\mathcal{P}_{n}=\bigcup_{k<n} P_{k} \neq \operatorname{supp} \mu$ for every $n \geq 1$. Thus there are arbitrarily large $n$ such that $\mu\left(\mathcal{P}_{n} \backslash \overline{\mathcal{P}}_{n-1}\right)>0$. Choose such a large $n$, and let $x \in \operatorname{supp} \mu \mid \mathcal{P}_{n} \backslash \mathcal{P}_{n-1}$. Then $x$ is a periodic orbit of period exactly $n$. We can approximate any $v \in C(X, \mathbb{R})$ by some $v^{\prime}$ which is constant in a compact neighborhood $K$ of $\left\{f^{k}(x)\right\}_{k=0}^{n-1}$. Moreover, we may assume that the set $\Sigma$ of $E \in \mathbb{R}$ such that $\left|\operatorname{tr} A_{n}^{\left(E-v^{\prime}\right)}(x)\right| \leq 2$ has exactly $n$ connected components: This is because the values $v^{\prime}\left(f^{k}(x)\right), 0 \leq k \leq n-1$, may be chosen independently and "the spectrum of the generic periodic Schrödinger operator has all gaps open" (indeed, when changing one of the values of $v^{\prime}\left(f^{n-1}(x)\right)$ keeping the others fixed, there are only at most $2 n$ exceptional values for which $\Sigma$ has less than $n$ connected components; see Claim 3.4 of [A2] for an argument). Under this assumption, the length of each connected component of $\Sigma$ is bounded by $2 \pi / n$ (see Lemma 2.4 of [A2]), so there exists $E \in(-3 \pi / n, 3 \pi / n)$ such that $\left|\operatorname{tr} A^{\left(E-v^{\prime}\right)}(x)\right|>2$. It follows that $L\left(E-v^{\prime}\right) \geq \mu\left(P_{n} \cap K\right) \gamma / n$, with $\gamma=\lim _{k \rightarrow \infty} \ln \left\|A_{k n}^{E-v^{\prime}}(x)\right\|^{1 / k}>0$.

Lemma 3.2. Let $v, v_{0}, w \in C(X, \mathbb{R})$ and let $\epsilon>0$. If $L(v+\epsilon w)>0$, then $\Phi_{\epsilon}\left(v, v_{0}, w\right)>0$.

Proof. By Lemma 2.3. $\gamma(t)=L\left(v+\epsilon\left(t v_{0}+\left(1-t^{2}\right) w\right)\right)$ is a subharmonic function in $\mathbb{C}$. 
By subharmonicity, if $\gamma(t)=0$ for almost every $t \in(-1,1)$, then $\gamma(t)=0$ for every $t \in(-1,1)$. So if $\gamma(0)=L(v+\epsilon w)>0$, we must have $\Phi_{\epsilon}\left(v, v_{0}, w\right)>0$ as well.

3.1. Proof of Theorem 2, We must show that for every $E \in C(X, \mathbb{R})$ and any non-empty open set $\mathcal{W} \subset V$, there exists $v_{2} \in W$ with $L\left(E-v_{2}\right)>0$.

Let $v_{1} \in \mathcal{W}$, let $v=E-v_{1}$ and let $v_{0} \in V$ be arbitrary with $\eta=\inf v_{0}>0$. Let $\epsilon>0$ be such that $v_{1}-\epsilon t v_{0} \in \mathcal{W}, t \in[-1,1]$. By Lemmas 3.1] and 3.2. there exists $w \in \mathcal{B}\left(\eta / 2^{3 / 2}\right)$ such that $\Phi_{\epsilon}\left(v, v_{0}, w\right)>0$. Since $V$ is dense in $C(X, \mathbb{R})$ and $w^{\prime} \mapsto \Phi_{\epsilon}\left(v, v_{0}, w^{\prime}\right)$ is continuous in $\mathcal{B}\left(\eta / 2^{3 / 2}\right)$ (by Theorem 6 ), we may assume that $w \in V$.

By Theorem 6, $\gamma(s)=\Phi_{\epsilon}\left(v, v_{0}, s w\right)$ is an analytic function of $s \in[-1,1]$. Since $\gamma(1)>0, \gamma(s)>0$ for every $s>0$ sufficiently small. Choose $s>0$ such that $v_{1}-\epsilon\left(t v_{0}+\left(1-t^{2}\right) s w\right) \in \mathcal{W}$ for every $t \in[-1,1]$ and such that $\gamma(s)>0$. Then there exists $t \in(-1,1)$ such that $L\left(v+\epsilon\left(t v_{0}+\left(1-t^{2}\right) s w\right)\right)>0$. Letting $v_{2}=$ $v_{1}-\epsilon\left(t v_{0}+\left(1-t^{2}\right) s w\right)$, we get $L\left(E-v_{2}\right)>0$ and $v_{2} \in \mathcal{W}$ as desired.

3.2. Proof of Theorem 1. The proof is readily adapted from the proof of Theorem 2] with the key Theorem [6] being replaced by Theorem 7 . once we provide appropriate corresponding statements to Lemmas 3.1 and 3.2 . The corresponding statement to Lemma 3.2 is that $L\left(e^{\epsilon a} A\right)>0$ implies that

$$
\int_{-1}^{1} \frac{1-t^{2}}{\left|t^{2}+2 i t+1\right|^{2}} L\left(e^{\epsilon\left(t b+\left(1-t^{2}\right) a\right)} A\right) d t>0
$$

and the proof is the same. The corresponding statement to Lemma 3.1 is that positive Lyapunov exponents are dense in $C(X, \mathrm{SL}(2, \mathbb{C}))$, and the argument goes along the same lines: If the set of non-periodic points has positive $\mu$-measure, then for a generic $A$ and almost every $\theta$ one has $L\left(R_{\theta} A\right)>0$ (see Remark 4.3 of [AD] ), and otherwise one uses that for a generic cocycle $A$ over a periodic orbit of period $n, R_{\theta} A$ is uniformly hyperbolic for an $O(1 / n)$ dense set of $\theta$.

\section{Prevalence of Density of positive Lyapunov exponents}

By an affine embedding of the Hilbert cube in a topological vector space $\mathcal{E}$ we shall understand a continuous affine map $D:[0,1]^{\mathbb{N}} \rightarrow \mathcal{E}$ (we do not assume injectivity). For such an affine embedding, the probability measure on $\mathcal{E}$ obtained by push forward of the product measure on $[0,1]^{\mathbb{N}}$ is denoted by $\rho_{D}$.

An affine embedding is called non-degenerate if its image is not contained in a proper closed affine subspace of $\mathcal{E}$. We call a Borel set $N \subset \mathcal{E}$ negligible if $\rho_{D}(N)=0$ for every non-degenerate affine embedding $D$.

It is clear that if $\mathcal{E}$ is a separable Banach space, then the complement of a negligible set $N$ is prevalent in $\mathcal{E}$, according to the definition given in the introduction.

Remark 4.1. In fact, it is possible to show that a negligible set in a separable Banach space is always a Gauss-null set; see [BL, Section 6.2]. The notion of Gauss-null sets is strictly stronger than that of Haar-null sets (i.e., the complement of a prevalent set) unless $\mathcal{E}$ is finite dimensional.

It is immediate from the definition that if $\mathcal{F} \subset \mathcal{E}$ is a dense subspace which is endowed with a finer topological vector space structure, then for any $\mathcal{E}$-negligible subset $N, \mathcal{F} \cap N$ is $\mathcal{F}$-negligible. Thus the following result contains Theorem 3 . 
Theorem 8. The set $N$ of all $v \in C(X, \mathbb{R})$ for which the set of energies $E$ with $L(E-v)>0$ is not dense in $\mathbb{R}$ is negligible.

The proof of this theorem will be based on an analysis of affine one-parameter families of potentials.

Theorem 9. Let $v \in C(X, \mathbb{R}), w \in \mathcal{B}\left(2^{-3 / 2}\right)$ and let $\epsilon>0$. If $L(-v-\epsilon w)>0$, then for almost every $t \in(0, \epsilon)$ there exists $E \in(-2 \epsilon, 2 \epsilon)$ such that $L(E-v-t w)>0$.

Before proving this result, let us see how it leads to Theorem 8 .

Proof of Theorem 8. Fix $\epsilon>0$, and let $N_{\epsilon}$ be the set of all $v \in C(X, \mathbb{R})$ such that $L(E-v)=0$ for every $E \in(-3 \epsilon, 3 \epsilon)$. Naturally $N=\bigcup_{q \in \mathbb{Q}} \bigcup_{n \in \mathbb{N}}\left(N_{2^{-n}}+q\right)$, so it is enough to show that $N_{\epsilon}$ is negligible for every $\epsilon>0$ (the translate of a negligible set is obviously negligible). Fix a non-degenerate embedding $D:[0,1]^{\mathbb{N}} \rightarrow C(X, \mathbb{R})$.

For $x \in[0,1]^{\mathbb{N}}$, let us denote by $S_{k}(x)$ the set of all $y \in[0,1]^{\mathbb{N}}$ with the same $k$ initial coordinates, and let $G_{k}$ be the tangent space to the smallest affine space containing $D\left(S_{k}(x)\right)$.

We claim that for every $x \in[0,1]^{\mathbb{N}}$, there exists $k(x) \in \mathbb{N}$ and a non-trivial segment $[x, y(x)] \subset S_{k}(x)$ such that $\Phi_{\epsilon}(-D(x),-D(x+t(y(x)-x))+D(x))>0$ for every $0<t \leq 1$. Indeed, if the conclusion does not hold, then $w \mapsto \Phi_{\epsilon}(-D(x), w)=$ 0 for every $w \in \bigcup_{k} G_{k}$ with $\|w\|<2^{-3 / 2}$ (by analyticity, Theorem 4 ). But $\bigcup_{k} G_{k}$ is dense by non-degeneracy, so by continuity of $w \mapsto \Phi_{\epsilon}(-D(x), w), \Phi_{\epsilon}(-D(x), w)=0$ for $w \in \mathcal{B}\left(2^{-3 / 2}\right)$. This contradicts Lemmas 3.1 and 3.2 .

Let $k(x)$ and $y(x)$ be as in the claim and let $w^{\prime}(x)=(-D(y(x))+D(x))$. Up to replacing $y(x)$ by $x+t(y(x)-x)$ for small $t>0$, we may assume that $\left\|w^{\prime}(x)\right\|<2^{-3 / 2}$. By continuity in $v$ of $\Phi_{\epsilon}\left(v, w^{\prime}(x)\right)$ (Theorem 6), there exists an open neighborhood $\mathcal{V}(x) \subset[0,1]^{\mathbb{N}}$ of $x$ such that $\Phi_{\epsilon}\left(-D(z), w^{\prime}(x)\right)>0$ for every $z \in \mathcal{V}(x)$. Thus for every $z \in \mathcal{V}(x)$, there exists $E_{0} \in(-\epsilon, \epsilon)$ and $0<t_{0}<\epsilon$ such that $L\left(-D(z)+E_{0}+t_{0} w^{\prime}(x)\right)>0$. By Theorem 9 (with $v=D(z)-E_{0}$ and $\left.w=-w^{\prime}(x) t_{0} / \epsilon\right)$, for almost every $0<t<t_{0}$, there exists $E \in(-3 \epsilon, 3 \epsilon)$ such that $L\left(-D(z)+E+t w^{\prime}(x)\right)>0$. Thus, for every $z \in \mathcal{V}(x)$ and for almost every $0<t<t_{0}$, if $z+t(y(x)-x) \in[0,1]^{\mathbb{N}}$, then $D(z+t(y(x)-x))=D(z)-t w^{\prime}(x) \notin N_{\epsilon}$, so that $D^{-1}\left(N_{\epsilon}\right) \cap \mathcal{V}(x)$ intersects each line parallel to $y(x)-x$ in a set of zero onedimensional Lebesgue measure.

By Fubini's theorem, for each $z^{\prime} \in[0,1]^{\mathbb{N}}, D^{-1}\left(N_{\epsilon}\right) \cap \mathcal{V}(x)$ intersects $S_{k}\left(z^{\prime}\right)$ in a set of zero $k$-dimensional Lebesgue measure. By Fubini's theorem again, $D^{-1}\left(N_{\epsilon}\right) \cap$ $\mathcal{V}(x)$ has zero (product) measure.

Since $[0,1]^{\mathbb{N}}$ is compact, we can cover it by finitely many $\mathcal{V}(x)$, and we conclude that for almost every $z \in[0,1]^{\mathbb{N}}, D(z) \notin N_{\epsilon}$, as desired.

The proof of Theorem 9 will involve some preparation. It will be convenient to consider a "convolved" variation of the functional $\Phi_{\epsilon}$. For $0<\delta<1$, let

$$
\Phi_{\epsilon, \delta}(v, w)=\int_{0}^{1} \int_{-\delta}^{\delta} \Phi_{\epsilon}(v+\epsilon a, b w) d a d b .
$$

Before getting into the details, let us explain the idea of the proof, in a simpler context. Suppose that we have a bounded, non-negative Borel function of two variables $h(x, y)$, and assume that there is a regularizing expression $H(y, s)=$ $\int_{0}^{1} \int_{-\epsilon}^{\epsilon} h(a, y+s b) d a d b$ defining a non-vanishing analytic function of $s$ which depends continuously on $y$. If $H(y, 0)=0$, then $H(y, s)$ is asymptotic to $c s^{k}$ for small $s$, 
where $c=c(y)>0$ and $k=k(y) \geq 2$ is an even integer. If there is a positive measure set $Z$ of $y$ with $H(y, 0)=0$, there is some $k$ such that $k(y)=k$ for $y$ in some compact set $K \subset Z$ of positive measure. Restricted to $K, c(y)$ is continuous. Since $K$ has positive measure, we can find two points $y, y+s \in K$ with $s>0$ arbitrarily small. It follows that $H(y, s)+H(y+s, s)<H(y, 2 s)$ (the left-hand side is like $2 c(y) s^{k}$ and the right-hand side is like $\left.2^{k} c(y) s^{k}\right)$. But this contradicts the trivial "covering estimate" $H(y, s)+H(y+s, s)=2 H(y, 2 s)$. Thus we must have $H(y, 0)>0$ for almost every $y$, which implies that $h(x, y)>0$ for a positive measure set of $x \in[-\epsilon, \epsilon]$.

We would like to apply this argument to the function $h(x, y)=L(v+x+y w)$, but the regularized expression we have (Theorem 4) is slightly more complicated. In particular it is not given by convolution with a bounded function, but this can be easily corrected by considering the convolved version (24). A covering estimate can then be obtained by bounding the convolution kernels (this is quite general and does not depend on analyticity):

Lemma 4.2. For every $0<\delta_{0}<\delta_{1}<1$, there exist $C_{1}, C_{2}>0$ such that for every $\kappa>0$, if $Z \subset(0,1)$ is a countable set such that for every $0<r_{0}<1$ there exists $r<r_{0}<(1+\kappa) r$ such that $r \in Z$, then for every $\epsilon>0$ and $v, w \in C(X, \mathbb{R})$ we have

$$
\Phi_{\epsilon, \delta_{0}}(v, w) \leq \sum_{r \in Z} C_{2} \kappa r \Phi_{\epsilon, \delta_{1}}\left(v+r \epsilon w, C_{1} \kappa r w\right) .
$$

Proof. Let $\Delta \subset \mathbb{R}^{2}$ be the set of all $(E, t)$ such that $0 \leq t \leq 1-E^{2}$. Let $\nu$ be the absolutely continuous measure supported on $\Delta$ with density $\frac{d \nu}{d E d t}=\frac{1}{\left|1+2 i E+E^{2}\right|^{2}}$, let $\gamma_{\delta}$ be the linear (one-dimensional Hausdorff) measure on $[-\delta, \delta] \times\{0\}$, and let $\nu_{\delta}=\nu * \gamma_{\delta}$. Let $T_{r}(E, t)=(E, t+r), S_{s}(E, t)=(E, s t)$ and $\nu_{\delta, r, s}=\left(T_{r}\right)_{*}\left(S_{s}\right)_{*} \nu_{\delta}$.

We can write

$$
\Phi_{\epsilon, \delta}(v+r \epsilon w, s w)=\int L(v+\epsilon(E+t w)) d \nu_{\delta, r, s}(E, t) .
$$

In order to establish the desired estimate, it is enough to show that

$$
\nu_{\delta_{0}} \leq \sum_{r \in Z} C_{2} \kappa r\left(T_{r}\right)_{*}\left(S_{C_{1} \kappa r}\right)_{*} \nu_{\delta_{1}}
$$

for appropriate choices of $C_{1}$ and $C_{2}$.

The measure $\nu$ has density bounded above by 1 and bounded below by $1 / 8$ through $\Delta$. Thus the measure $\nu_{\delta_{0}}$, whose support is contained in the rectangle $\left[-1-\delta_{0}, 1+\delta_{0}\right] \times[0,1]$, has density bounded by $2 \delta_{0}$. On the other hand, there exists $C_{1}=C_{1}\left(\delta_{0}, \delta_{1}\right) \geq 1$ such that the density of $\mu_{\delta_{1}}$ is at least $\frac{\delta_{1}-\delta_{0}}{10}$ in the rectangle $\left[-1-\delta_{0}, 1+\delta_{0}\right] \times\left[0, C_{1}^{-1}\right]$. So $C_{1} \kappa r\left(T_{r}\right)_{*}\left(S_{C_{1} \kappa r}\right)_{*} \nu_{\delta_{1}}$ has density at least $\frac{\delta_{1}-\delta_{0}}{10}$ in the rectangle $\mathcal{R}_{r}=\left[-1-\delta_{0}, 1+\delta_{0}\right] \times[r,(1+\kappa) r]$. Letting $C_{2}=20 \frac{\delta_{0}}{\delta_{1}-\delta_{0}} C_{1}$, we see that the right-hand side of (27) has density at least $2 \delta_{0}$ in $\bigcup_{r \in Z} \mathcal{R}_{r}$, which by hypothesis contains $\left[-1-\delta_{0}, 1+\delta_{0}\right] \times(0,1)$. The result follows.

The next lemma uses in a simple way the analyticity of $\Phi_{\epsilon}$, as well as continuity with respect to parameters to get the desired asymptotic expansions. 
Lemma 4.3. Let $\epsilon>0, v, w \in C(X, \mathbb{R})$ be such that

1. $t \mapsto \Phi_{\epsilon}(v, t w)$ is non-identically vanishing near $t=0$,

2. for every $\xi>0$, the set $X_{\xi}$ of $t \in[-\xi, \xi]$ such that $L(v+t w+E)=0$ for every $E \in[-2 \epsilon, 2 \epsilon]$ has positive Lebesgue measure.

Then for every $\xi>0$, there exists $C>0$, a compact set $K \subset[-\xi, \xi]$ of positive Lebesgue measure, a non-trivial closed interval $J \subset[-\epsilon, \epsilon]$, a continuous function $c: J \times K \rightarrow \mathbb{R}_{+}$, and an even integer $k \geq 2$, such that for every $(E, t) \in J \times K$, we have

$$
\left|\Phi_{\epsilon}(v+E+t w, s w)-c(E, t) s^{k}\right| \leq C s^{k+1}, \quad|s| \leq 1 .
$$

Proof. The function

$$
s \mapsto \Phi_{\epsilon}(v+E+t w, s w)
$$

is analytic in a neighborhood of $s=0$ for each $(E, t) \in \mathbb{R}^{2}$ and depends continuously on $(E, t)$ (as an analytic function). At $(E, t)=(0,0)$, this function is non-identically vanishing. Select $0<\xi<\epsilon$ so that non-vanishing holds for $(E, t) \in[-\xi, \xi]^{2}$. At each $(E, t) \in[-\epsilon, \epsilon] \times X_{\xi}$ we have $\Phi_{\epsilon}(v+E+t w, 0)=0$.

By the non-vanishing and analyticity of (29), for each $(E, t) \in[-\xi, \xi] \times X_{\xi}$ there exists an even integer $k(E, t) \geq 2$ and $c(E, t)>0$ such that $\Phi_{\epsilon}(v+E+t w, s w)=$ $c(E, t) s^{k(E, t)}+O\left(s^{k(E, t)+1}\right)$. Using also the continuity (as an analytic function) of (29) with respect to $(E, t)$, we see that $k(E, t)$ is an upper-semicontinuous function. Let $W_{\xi} \subset X_{\xi}$ be the set of Lebesgue density points, and for $l \geq 2$ even, let $M_{\xi}^{l} \subset$ $[-\xi, \xi] \times W_{\xi}$ be the set of $(E, t)$ with $k(E, t)=l$. Let $k_{0}$ be minimal such that $M_{\xi}^{k_{0}}$ is non-empty. By upper-semicontinuity, $M_{\xi}^{k_{0}}$ is open in $[-\xi, \xi] \times W_{\xi}$ so it has positive Lebesgue measure.

Let $\left(E_{0}, t_{0}\right) \in M_{\xi}^{k_{0}}$, and let $\delta>0$ be such that if $(E, t) \in[-\xi, \xi] \times W_{\xi}$ and $\left|t-t_{0}\right| \leq \delta,\left|E-E_{0}\right| \leq \delta$, then $(E, t) \in M_{\xi}^{k_{0}}$. Let $K \subset W_{\xi} \cap\left[t_{0}-\delta, t_{0}+\delta\right]$ be a compact set of positive Lebesgue measure and let $J=[-\xi, \xi] \cap\left[E_{0}-\delta, E_{0}+\delta\right]$. Then for $(E, t) \in J \times K, \Phi_{\epsilon}(v+E+t w, s w)=c(E, t) s^{k}+O\left(s^{k+1}\right)$. By continuity (as an analytic function) of (29) with respect to $(E, t)$, the function $c(E, t)$ is continuous over $J \times K$ and the error term is uniform over $J \times K$.

We now combine the two estimates to get a result by contradiction.

Lemma 4.4. Let $\epsilon>0, v, w \in C(X, \mathbb{R})$ be such that $t \mapsto \Phi_{\epsilon}(v, t w)$ is nonidentically vanishing near $t=0$. Then for almost every small $t$, there exists $E \in[-2 \epsilon, 2 \epsilon]$ with $L(v+E+t w)>0$.

Proof. If the result did not hold, then $\epsilon, v, w$ satisfy the hypothesis of the previous lemma. Let $J, K, C, c$ and $k$ be as in the previous lemma, let $t_{1}$ be a Lebesgue density point of $K$, and let $E_{1}$ be the center of $J$. Let $v_{1}=v+E_{1}+t_{1} w$, and let $0<\delta_{0}<\delta_{1}<\min \{1,|J| / 2\}$. It follows that if $c_{1}>0$ is sufficiently small $\left(c_{1}<\frac{1}{k+1} \int_{-\delta_{0}}^{\delta_{0}} c\left(E_{1}+a, t_{1}\right) d a\right)$, then for every $s>0$ sufficiently small,

$$
\Phi_{\epsilon, \delta_{0}}\left(v_{1}, s w\right)>c_{1} s^{k} .
$$

On the other hand, if $c_{2}>0$ is sufficiently large, then for every $s>0$ sufficiently small, if $t \in K$, then

$$
\Phi_{\epsilon, \delta_{1}}\left(v+E_{1}+t w, s w\right) \leq c_{2} s^{k} .
$$

Fix such constants $c_{1}$ and $c_{2}$, and let $C_{1}$ and $C_{2}$ be the constants of Lemma 4.2. 
Since $t_{1}$ is a Lebesgue density point of $K$, for every $\kappa>0$ we can select a sequence $s_{i}>0, i \geq 1$, such that $t_{1}+\epsilon s_{i} \in K$ and $\kappa / 2<\frac{s_{i}}{s_{i+1}}-1<\kappa$.

Let $s>0$ be small and let $i_{0}>0$ be minimal with $s_{i}<s$. By Lemma 4.2. we get

$$
\Phi_{\epsilon, \delta_{0}}\left(v_{1}, s w\right) \leq \sum_{i \geq i_{0}} C_{2} s_{i} s^{-1} \kappa \Phi_{\epsilon, \delta_{1}}\left(v_{1}+\epsilon s_{i} w, C_{1} \kappa s_{i} w\right)
$$

This gives

$$
c_{1} s^{k}<\sum_{i \geq i_{0}} C_{2} s_{i} s^{-1} \kappa c_{2}\left(C_{1} \kappa s_{i}\right)^{k}
$$

that is,

$$
c_{1} C_{2}^{-1} c_{2}^{-1} C_{1}^{-k} \kappa^{-k-1}<\sum_{i \geq i_{0}}\left(\frac{s_{i}}{s}\right)^{k+1} .
$$

On the other hand, clearly

$$
\sum_{i \geq i_{0}}\left(\frac{s_{i}}{s}\right)^{k+1} \leq \sum_{j \geq 0}\left(1-\frac{\kappa}{2}\right)^{j(k+1)} \leq 2 \kappa^{-1}
$$

which contradicts (34) for small $\kappa>0$.

Proof of Theorem 9. By Theorem 4, for every $t \in(0,1), s \mapsto \Phi_{\epsilon}(-v-t \epsilon w,-s(1-$ $t) w)>0$ is an analytic function of $s \in[-1,1]$, and by Lemma 3.2 and the hypothesis, it is non-zero at $s=1$. By Lemma 4.4 for every $t \in(0,1)$, for almost every small $s$, there exists $E \in(-2 \epsilon, 2 \epsilon)$ such that $L(E-v-\epsilon(t+s(1-t)) w)>0$. The result follows.

\section{REFERENCES}

[A1] Avila, A., Density of positive Lyapunov exponents for quasiperiodic SL $(2, \mathbb{R})$ cocycles in arbitrary dimension. Journal of Modern Dynamics 3 (2009), 629-634. MR2587090

[A2] Avila, A., On the spectrum and Lyapunov exponent of limit periodic Schrödinger operators. Comm. Math. Phys. 288 (2009), 907-918. MR2504859 (2010f:47057)

[A3] Avila, A., On the Kotani-Last and the Schrödinger Conjectures. In preparation.

[AB] Avila, A.; Bochi, J., A formula with some applications to the theory of Lyapunov exponents. Israel Journal of Mathematics 131 (2002), 125-137. MR.1942304 (2004a:37036)

[AD] Avila, A.; Damanik, D., Generic singular spectrum for ergodic Schrödinger operators. Duke Math. J. 130 (2005), 393-400. MR2181094 (2006k:82083)

[AK] Avila, A.; Krikorian, R., Monotonic cocycles. In preparation.

[AMS] Avron, J.; van Mouche, P. H. M.; Simon, B., On the measure of the spectrum for the almost Mathieu operator. Comm. Math. Phys. 132 (1990), no. 1, 103-118. MR.1069202 (92d:39014a)

[AS] Avron, J.; Simon, B., Almost periodic Schrödinger operators. II. The integrated density of states. Duke Math. J. 50 (1983), 369-391. MR700145 (85i:34009a)

[BL] Benyamini, Y.; Lindenstrauss, J., Geometric non-linear functional analysis, Volume 1, Colloquium Publication, No. 48, American Mathematical Society, Providence, 1999. MR:1727673 (2001b:46001)

[B] Bochi, J., Genericity of zero Lyapunov exponents. Ergodic Theory and Dynamical Systems 22 (2002), 1667-1696. MR.1944399 (2003m:37035)

[BV] Bochi, J.; Viana, M., Lyapunov exponents: How frequently are dynamical systems hyperbolic? Modern dynamical systems and applications, 271-297, Brin, Hasselblatt, Pesin (eds.), Cambridge Univ. Press, 2004. MR2090775 (2005g:37060)

[BGV] Bonatti, C.; Gómez-Mont, X.; Viana, M., Généricité d'exposants de Lyapunov non-nuls pour des produits déterministes de matrices. Ann. Inst. H. Poincaré Anal. Non Linéaire 20 (2003), 579-624. MR1981401 (2004f:37041) 
[D] Damanik, D., Lyapunov exponents and spectral analysis of ergodic Schrödinger operators: a survey of Kotani theory and its applications. Simon Festschrift, Proceeding of Symposia in Pure Mathematics, American Mathematical Society, Providence. MR 2307747 (2008c:82040)

[DS] Dinaburg, E. I.; Sinai, Ja. G., The one-dimensional Schrödinger equation with quasiperiodic potential. Funkcional. Anal. i Prilozen. 9 (1975), no. 4, 8-21. MR0470318 (57:10076)

[FK] Fayad, B.; Krikorian, R., Rigidity results for $\mathrm{SL}(2, \mathbb{R})$ cocycles above rotations of the circle. Journal of Modern Dynamics 3 (2009), 479-510. MR2587083

[HSY] Hunt, B.; Sauer, T.; Yorke, J., Prevalence: a translation-invariant "almost every" on infinite-dimensional spaces. Bull. Amer. Math. Soc. (N.S.) 27 (1992), no. 2, 217-238. MR $1161274(93 \mathrm{k}: 28018)$

[J] Jitomirskaya, S., Ergodic Schrödinger operators (on one foot). Simon Festschrift, Proceeding of Symposia in Pure Mathematics, American Mathematical Society, Providence. MR 2307750(2008g:82055)

[Ka] Katok, A., Bernoulli diffeomorphisms of surfaces. Ann. Math. 110 (1979), 529-547. MR.554383 (81a:28015)

[Kol] Kolmogorov, A. N., The general theory of dynamical systems and classical mechanics. Proceedings of the International Congress of Mathematicians (Amsterdam, 1954), Vol. 1, pages 315-333, North Holland, Amsterdam, 1957. MR0097598 (20:4066)

[K] Kotani, S., Ljapunov indices determine absolutely continuous spectra of stationary random one-dimensional Schrödinger operators. Stochastic Analysis (Katata/Kyoto, 1982), 225-247, North-Holland Math. Library 32, North-Holland, Amsterdam, 1984. MR780760 (86h:60117)

[Y] Yoccoz, J.-C., Some questions and remarks about SL(2, $\mathbb{R})$ cocycles. In Modern Dynamical Systems and Applications, 447-458. Cambridge University Press, Cambridge, 2004. MR2093316 (2005h:37068)

[V] Viana, M., Almost all cocycles over any hyperbolic system have non-vanishing Lyapunov exponents. Ann. Math. 167 (2008), 643-680. MR.2415384(2009i:37080)

Institut de Mathématiques de Jussieu, CNRS UMR 7586, 175 Rue du Chevaleret, 75013, PARIs, France

IMPA, Estrada Dona Castorina 110, 22460-320, Rio de Janeiro, Brazil

$U R L$ : www.impa.br/ avila/

E-mail address: artur@math.sunysb.edu 